\title{
TEXTRONIC SOLUTIONS USED FOR PREMATURE BABIES: A REVIEW
}

\author{
Karolina Wilgocka ${ }^{1,2, *}$, Ewa Skrzetuska1, Izabella Krucińska1, Witold Sujka² \\ 1 Technical University of Lodz, Institute of Materials Science of Textiles and Polymer Composites, Lodz, Poland \\ 2 TRICOMED SA, Świętojańska 5/9, 93-493 Lodz, Poland \\ ${ }^{*}$ Corresponding author. E-mail: karolina.wilgocka@tzmo-global.com
}

\begin{abstract}
:
Premature birth is considered to be a substantial problem in perinatal medicine, which in the vast majority of cases (>60\%), concerns African and South Asian countries. Nevertheless, prematurity is a global problem and is faced by both less-developed (where $12 \%$ of babies are prematurely born) and well-developed countries (with $9 \%$ prematurity rate) $[1,2]$. The percentage of children born prematurely, i.e., before the 37 th week of pregnancy, was $8.7 \%$ in Europe, while, in Poland, it was 7.34\% [3]. Care of prematurely born babies is a huge challenge for parents and medical staff in the neonatal intensive care unit. Preterm infants, because of their low weight and gestational age, are prone to health problems and even death. For this reason, continuous monitoring of health parameters plays an important role. It is achieved by the use of various sensors that are inserted in infants' garments. Sensor systems monitor an infant's health condition, and then the data are transmitted to doctors or parents. This article is for illustrative purposes, aimed at presenting solutions such as the use of sensors for monitoring infants' physiological parameters.
\end{abstract}

\section{Keywords:}

Wearable sensors for infants, textronics, smart clothing, health monitoring, neonatal monitoring, wearable sensors

\section{Introduction}

Currently, technological development and the pursuit of miniaturization of electronic devices have given the opportunity to develop new types of textronic materials with a wide range of applications in the automotive industry, sports, and medicine. The combination of various fields of science has allowed the creation of innovative textiles with novel applications, which are thus often more desirable than traditional materials [4].

Textronic is an interdisciplinary field of science that integrates electronics, textiles, information technology (IT), metrology, and automation. It is based on the approach of projecting and manufacturing textile fabrics. Textronic solutions are born through the synergy of a couple of sciences and lead to multifunctional materials, or the so-called intelligent materials $[5,6]$.

Textronic development has contributed to the creation of specific sensors for monitoring life parameters used in everyday-wear garments. The use of garment sensors establishing, e.g., respiration rhythm, enables us to detect anomalies at the initial stages of sleep apnea in infants, or electrocardiogram (ECG) sensors permit the detection of abnormal heart rhythms [7].

Detecting irregularities in a baby's condition is crucial because his/her systems are not yet fully developed [8]. Multiparameter monitoring provides complex, safe, reliable, and precise health care, especially for premature babies in the intensive care unit
(ICU) [9]. Newborn babies in the ICU require special treatment, the quality of which conditions the baby's further development. The smaller and younger the baby is, the greater is the risk of life threat or death rate [10]. Disorders in organ functioning are common in prematurely born babies because their central nervous system, which is responsible for thermoregulation and respiration, is not fully mature.

According to the World Health Organization (WHO), babies are considered premature when they are born between the 22nd and 37th weeks of pregnancy. Children who are born before the 28th week of pregnancy are called extremely premature. The premature baby's weight, in the vast majority of cases, is $<2,500 \mathrm{~g}$. There have been cases in which the premature baby was $<1,000 \mathrm{~g}$ in weight. Infants born before the due date do not have a well-developed respiratory, central nervous, or cardiovascular system. The basic parameters that need to be controlled are heart rate (HR), breath, pulse, saturation, and humidity $[1,10,11]$.

Maintaining an appropriate environment for the premature baby's development becomes a challenge for medical staff. It is difficult to provide the thermal environment for the infant. Hypothermia in premature babies within the first 12 hours of life is a factor determining mortality and susceptibility to illness. WHO recommends that premature babies' temperatures should be in the range of $36.5-37.5^{\circ} \mathrm{C}$. Temperatures of $36.0-36.4^{\circ} \mathrm{C}$ indicate mild hypothermia, $32.0-35.9^{\circ} \mathrm{C}$ indicate moderate hypothermia, while temperatures $<32.0^{\circ} \mathrm{C}$ indicate 
severe hypothermia [12]. Newborn babies are prone to hypothermia because the relationship between surface area and mass/weight is high, and it increases together with loss in weight. Infants have relatively big heads, which easily lose heat through evaporation, because their skin is not mature yet. Thermal insulation is distorted because of little fat, which is an insulation barrier. A premature baby's skin cannot act as a mechanical barrier, which usually protects the baby from excessive water loss and acts as a thermoregulator, and as a result, the premature baby is susceptible to external environmental changes.

Because of the lack of stratum corneum in premature babies, the skin has the tendency to show transepidermal water loss (TEWL), which results in decrease in body heat. Such a condition of the infant leads to functional and metabolic disorders [7]. Premature babies' garments are therefore worth considering because they would provide physiological comfort and an optimal environment for the infants' development. Premature babies' garments may contain compatible sensors in their structures, which monitor the babies' life parameters.

Wearable sensors are applied in health monitoring, especially for the elderly and infants. Recently, immense progress in sensor systems, wireless communication, and supply technology has been observed, which has resulted in the creation of new-generation monitoring systems for premature babies. Integrated sensor systems for ECG, respiration, temperature, and humidity enable the detection of unwanted life-threatening events [9].

\section{Literature analysis}

The activity of the sensors used for monitoring life parameters is based on general rules of detection and processing of the collected data. Changes in life parameters may indicate potential changes in the organs, which is why, they should be monitored and, at the right moment, preventive measures should be implemented [9].

Assessment of the stability of cardiovascular and respiratory systems in premature babies is part of the basic care process in the ICU. During the entire period of stay in the hospital, the frequencies of the infant's life activities, such as HR, respiratory rate, and breathing frequency, are constantly monitored. Blood pressure is observed within the first days after birth and in case of severe illness. Another important parameter that is monitored is the temperature; however, its measures may be distorted by external heat sources [13].

The most frequently monitored vital functions of infants in intensive care are shown in Table 1.

Wearable systems for monitoring life parameters may contain many elements: sensors, intelligent materials, actuators, power supply, modules and wireless networks, steering and processing units, user interface, software, and advanced algorithms for data extraction. Sensor systems used for health monitoring must meet established functions, which are compliant with medical criteria and consider the limitations in the devices. The appearance should meet aesthetic expectations; the system's weight and size should be small and should not obstruct movement, especially of infants [9].

\subsection{Temperature sensors}

Newborn's body temperature is one of the most important parameters that require constant monitoring. Temperature acts as a signal of an infection and the general stability of the infant. The temperature is generally measured at one point on the body. Moreover, this parameter is used for establishing the

Table 1. The most important vital parameters that are monitored during neonatal intensive care.

\begin{tabular}{|c|c|c|c|}
\hline $\begin{array}{c}\text { Physiological } \\
\text { parameters }\end{array}$ & \multicolumn{2}{|c|}{ Principle of detection } & Transducers \\
\hline \multirow{4}{*}{ Temperature [9] } & \multirow{2}{*}{ Electrical } & Resistance & Thermistor \\
\hline & & Thermoelectric effect & Thermocouple \\
\hline & \multirow{2}{*}{ Optical } & Infrared emission & Infrared pyroelectric sensor \\
\hline & & Fluorescence & Photodetector \\
\hline \multirow{2}{*}{ Pulse and heart rate [9] } & \multirow{2}{*}{ Optical } & \multirow{2}{*}{ Pressure } & Force-sensitive resistor \\
\hline & & & Light-sensitive detector \\
\hline ECG and EEG [9] & Electrical & Biopotential & Metal or textile electrodes \\
\hline \multirow{2}{*}{ Respiration [9] } & Electrical & Impedance & Skin electrodes \\
\hline & Mechanical & Extension & Strain gauge \\
\hline \multirow{2}{*}{ Humidity [14] } & Resistive & $\begin{array}{l}\text { Changes in the electrical } \\
\text { impedance }\end{array}$ & $\begin{array}{l}\text { Precious-metal electrodes deposited on a glass or } \\
\text { ceramic substrate }\end{array}$ \\
\hline & Capacitive & $\begin{array}{l}\text { Changes in the dielectric } \\
\text { constant }\end{array}$ & $\begin{array}{l}\text { Electrodes (conductive plates) and a dielectric } \\
\text { material placed between the plates }\end{array}$ \\
\hline
\end{tabular}

EEG, electroencephalogram. 
appropriate temperature of the surroundings for optimal growth and child's development. Both electronic and optical devices can be used for temperature measurement [15].

Chen et al. [15] proposed a project that involved noninvasive temperature monitoring of an infant's body, in which wearable sensors were used. A thermistor was applied (negative temperature coefficient [NTC] sensor) as a temperature sensor because of its small size and precise measurement. Textile fibers integrate the sensor with monitoring platforms. The sensors' localization and the material with which the sensors are made are selected to ensure that their look is optimized for their functionality and infants' comfort.

The prototype belt was made of soft bamboo fibers with an integrated NTC sensor. An NTC Mon-A-Therm 90045 (2 mm $\times 3 \mathrm{~mm}$ ) temperature sensor was built into the belt. Elastic and soft textile fibers were made of silver-plated nylon yarn Shieldex ${ }^{\circledR}$. Silver yarns were woven in the material as flexible wires. The connection between the temperature sensor and the conductive wires was achieved by tying Shieldex ${ }^{\circledR}$ yarn on the sensor. Fixing the NTC sensor to the prototype belt was performed by stitching the sensor to the belt. To avoid contact between the textile cords, each wire was stitched at several places on the belt.

While designing the belt for body temperature measurements, researchers took into consideration the functional and technical aspects that the belt must comply with. The most prominent considerations were to provide continuous and precise temperature monitoring of the infant's body temperature inside the incubator, safety of use while in the ICU, ensuring of infant's comfort by avoiding both adhesive parts and contact of sharp edges with the infant's body, and, finally, ease in sensor cleaning and disinfection. Precision of the sensor used for monitoring the infant's body temperature should be $0.1^{\circ} \mathrm{C}$. Researchers placed the sensor above the liver because this organ is one of the most active ones, and the temperature in this area is higher. An increase in temperature may indicate developing infection.

The temperature sensor was insulated with soft cotton foam in order to limit the influence of external factors (temperature of the surroundings) on the measurements. In the designed belt prototype, no adhesive parts and rigid elements were used; elastic and soft textile fibers were applied instead as connectors. Additionally, the belt placement does not obstruct other measurements and routine newborn clinical treatment. Furthermore, the belt can be used in a complex monitoring system of all crucial newborn parameters: ECG, respiration rate, and peripheral oxygen saturation level $\left(\mathrm{SpO}_{2}\right)$.

The applied sensor must be calibrated first, and the next step is to connect the belt to a digital oscilloscope, so that data collection and temperature measurements would be displayed. Figure 1 illustrates the scheme of the setup used as a temperature monitoring system.

In order to establish the resistance, measurements were taken at various temperatures $\left(25^{\circ} \mathrm{C}-43^{\circ} \mathrm{C}\right)$. The sensor was tested in connection with both traditional and textile cords. On the basis of five measurements for each cord, an equation was introduced, and the temperature was measured based on the sensor's resistance value. It was observed that the higher the temperature, the lower is the sensor resistance, and that the textile cord's resistance is slightly above that of traditional ones.

The material used has an impact on the comfort, for which the infant's body temperature is measured. Apart from comfort, the material should be safe and breathable. Various materials were considered for prototype application. Attention was paid to textile features and ecological aspects. The material selected for the prototype belt was bamboo fabric, which is characterized by antibacterial properties that no other material has (e.g., linen, wool). Bamboo fabric is soft and provides appropriate air flow, which makes it comfortable to wear.

In order to verify the proper functioning of the prototype belt, a test was conducted on children in an incubator in the ICU. The prototype belt was tested on an infant born before the 30th week of pregnancy whose condition was stable. In the ICU, a neonatologist and a nurse were present during the examination. Once parents' agreement was obtained, the prototype belt was placed around the infant's body. The belt was tested for 45 minutes in order to check whether the signals from the belt remained stable for a longer time when the baby was wearing the belt. Results obtained from the experiment in the ICU indicated that the device may be safely used. In comparison to a monitor for a standard patient, the prototype belt provides precise temperature monitoring, and the monitoring error is within $0.1^{\circ} \mathrm{C}[15]$.

In a proposed solution for a prototype garment containing respiratory and temperature sensors, developed for infants, Jakubas et al. [8], used two types of temperature sensors, namely, analog and digital sensors. The children's garment equipped with sensors and electronic components is shown in Figure 2.
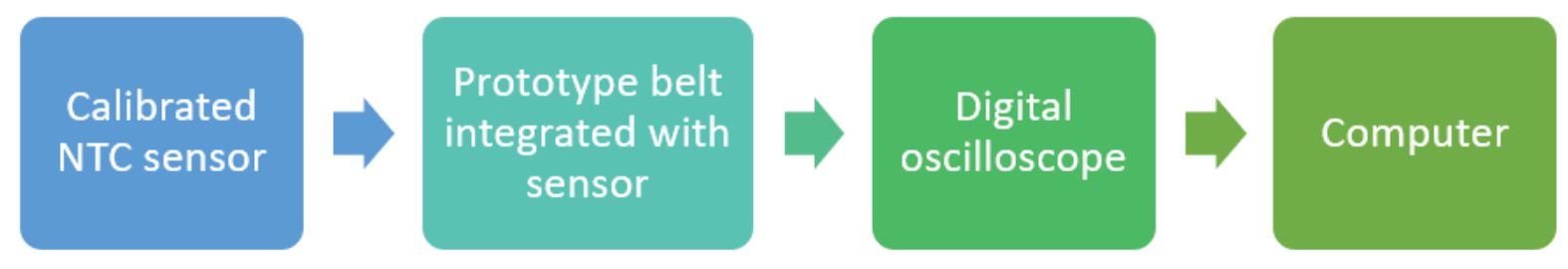

Figure 1. Scheme of the setup used as a temperature monitoring system. 


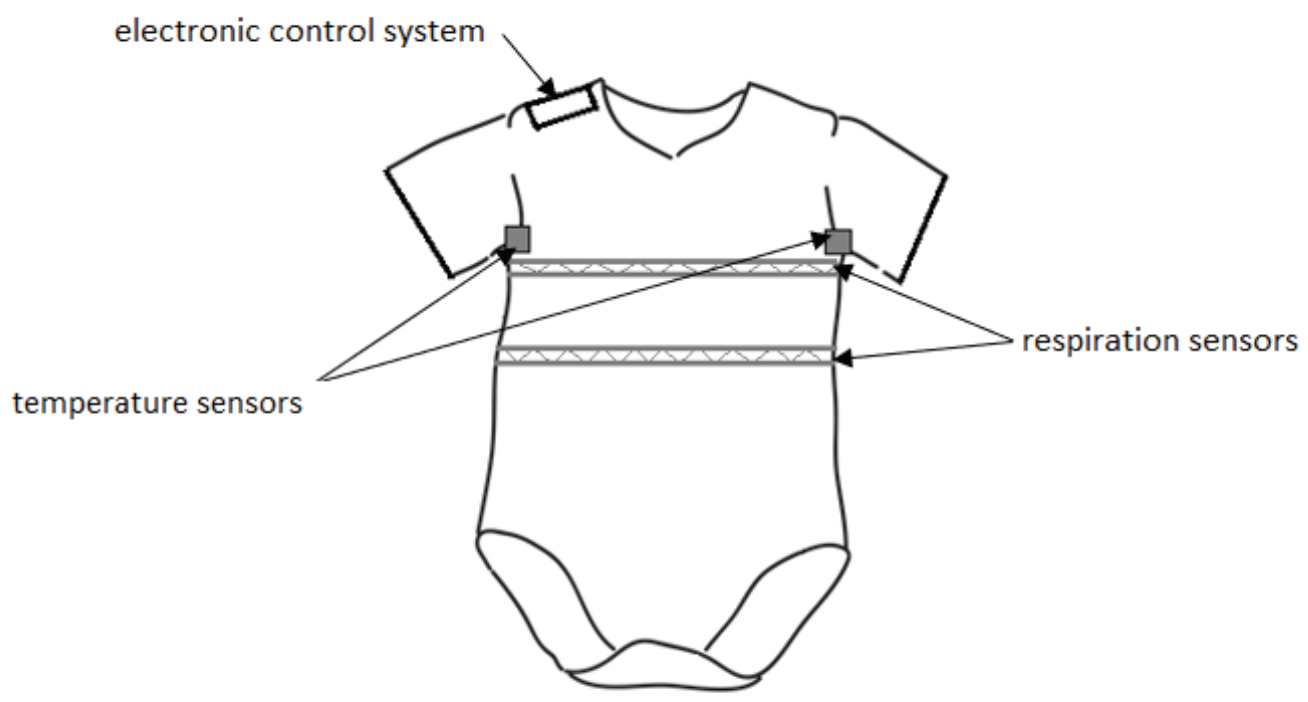

Figure 2. Children's garment equipped with sensors and electronic components.

The analog temperature sensor MCP9700 is characterized by small size and a linear change of output voltage in proportion to the temperature change $\left(10 \mathrm{mV} /{ }^{\circ} \mathrm{C}\right)$ and very low power consumption $(12 \mu \mathrm{A})$. The second sensor was TMP006. This temperature sensor works at a distance; therefore, in order to measure the temperature, it uses the infrared (IR) radiation emitted by a tested object. An interintegrated circuit $\left(\mathrm{I}^{2} \mathrm{C}\right)$ interface (intermediary between integrated circuits) was used for communication with the control system. All electronic components and the respiratory rhythm sensor were connected by textile wires manufactured using electroconductive yarns.

Contrasting problems were met depending on the type of sensor used. In the case of the analog sensor, a voltage drop on the signal line was caused by a change in line resistance due to the baby's movements, which affected measurement accuracy, whereas, in the case of electric sensors, the distortion was caused by clothing electrification [8].

\subsection{Respiration sensors}

An important parameter that demands constant control is also the child's breath. A dangerous ailment for newborns is sleep apnea, which leads to cot death, or sudden infant death syndrome (SIDS), which is the result of immature respiratory tract or organ infection.

In currently used intelligent garments, breath is measured on the basis of resistance control, which uses the piezoelectric effect or optical fibers. Such a sensor is integrated into the garment, or it is applied in the form of a band on the infant's chest. Sensors should provide accurate and repetitive measurements. Additionally, sensors should be resistant to factors, such as perspiration, bending, humidity, or chemical substances [8].

Many ready-to-use sensors are available on the market and can be used in solutions devoted to preterm infants. For instance,
$\mathrm{CO}_{2}$ sensors have various detection methods: electrochemical, IR sensors, or sensors using metal oxides. Electrochemical sensors provide better performance but short life service; IR sensors are characterized by high sensitivity but are rather large and expensive. Metal oxide sensors are low-cost sensors but susceptible to temperature and humidity [9].

In the study by Jakubas et al. [8], the garment uses the sensors of electrically conductive fibers. The infant's garment had respiratory sensors in the form of a belt, which contained textile sensors placed on the chest. An electronic system was an additional element. All electronic components and respiratory sensors were connected by textile power and signal lines built on the basis of electrically conductive threads.

Preliminary measurements were taken to verify the created textile sensor and to evaluate the 3-month-old baby's respiratory rhythm. Resistance measurement was based on chest moves while breathing. Relative measurements of the resistance sensor were conducted for two 5-minute periods. The obtained results (29-38 inhalations), correspond to medical data: $30+/-8$ inhalations per minute.

Hung Cao et al. [16] proposed the monitoring system for children to minimize the risk of SIDS. This system can be applied at home and in the hospital. It consists of a $\mathrm{CO}_{2}$ sensor and radio frequency identification technology (RFID). The commercial $\mathrm{CO}_{2}$ sensor was used based on metal oxide, characterized with reference to sensitivity, selectivity, and humidity. RFID transmission was achieved using wireless modules at two different operating frequencies. The set of sensors was placed on the bars of the bed in which the baby lies and connected to the processing board. The printed board used for data processing was placed outside the crib. It contained a wireless module for receiving and transmitting data. The wireless transmitter was located at the output of the analog multiplexer. The receiver split the signals using a demultiplexer to monitor multimodality. The battery was the power source. A radical change in $\mathrm{CO}_{2}$ 
concentration will cause a sharp change in the output signals of the sensor, and subsequently, the processor will be triggered to send an alarm signal.

The sensor circuit has an amplifier that works in two stages. The first stage of the amplifier is used for buffering, and a differential amplifier is located behind it. An analog multiplexer or AND gate is used to multiplex the signal. An alarm is sent if the sensor outputs are lower than the threshold value. The transmitter sends the data collected by the sensor and the alarm signal to the server.

A test was performed, involving breathing near the sensor at a distance of $50 \mathrm{~cm}$ for 30 seconds and then holding the breath for 20 seconds. Various breath directions and head positions were taken into account. The receiver was located at various distances and in different rooms. The light-emitting diode (LED) lit up just a couple of seconds after the test started. The analysis was repeated with two people using two sets of sensors/transmitters. The results were identical [16].

The system of respiratory rate monitoring, based on thermal sensors, was developed by Cheung-Hwa Hsu et al. [17] and is an example of noncontact respiratory monitoring. An apnea monitor is used for the detection and display of an infant's inhaling frequency using thermal sensors. The device registers temperature changes while breathing and displays them on the monitor. Such a setup does not evoke any irritation on the infant's body because there is no contact with the skin. Thermal sensors on the mask are sensitive to temperature changes caused by breathing, and the sensor data are gathered and analyzed by the computer connected to the central nursery. Owing to the use of a matrix of thermosensors, the mask has the ability to detect temperature changes induced by breathing. An ellipsoidal shape was used to avoid the lack of signal detection Thermosensors on the mask detect changes in the temperature of the air exhaled by the baby. Then the changes are magnified by a preamplifier. Sensitivity is adjustable through the use of a boost amplifier. Through an analog-to-digital (A/D) converter, the signal is sent to the microcontroller unit (MCU), which has the task of linking, controlling, and communicating between the subsystems. Afterward, the signal received and the data on physiological parameters are sent to a computer. Figure 3 illustrates a block diagram of the system [17].

\subsection{ECG sensors}

One of the main causes of incubator discomfort for an infant is the monitoring system. Crucial parameters are obtained through adhesive sensors placed on the delicate skin with a single wire connected to external monitors. Sensor placement and tangled cables lead to infants' discomfort, skin irritation, and sleep interruption. For this reason, a noninvasive alternative for monitoring a preterm infant's life parameters is on demand [18]. Taking into account possible skin damage, the use of traditional, gel electrodes in ECG measurement is not recommended. The development of textile electrodes, as well as progress in conductive fibers and fibers coated with metal and elastic electronics, makes biosignal measurements more effective and human-friendly [9, 19].

Sibrecht Bouwstra et al. [18] proposed a solution for the monitoring of infants' life parameters, namely, the Smart Jacket project. The Smart Jacket is useful for discrete and constant monitoring achieved through a network of sensors and wireless communication. Their design is the first version of a babies' jacket that enables ECG measurements using textile electrodes. The design of the Smart Jacket aims to provide continuous monitoring of the vital functions of a neonate while in an incubator or during Kangaroo mother care.

The prototype of the jacket has an open structure of the material on the front, back, and on the cup, thus making it easier for medical personnel to access the child. In order to maintain the structure of traditional children's clothing, a combination of white and green colors with animal figures was selected.

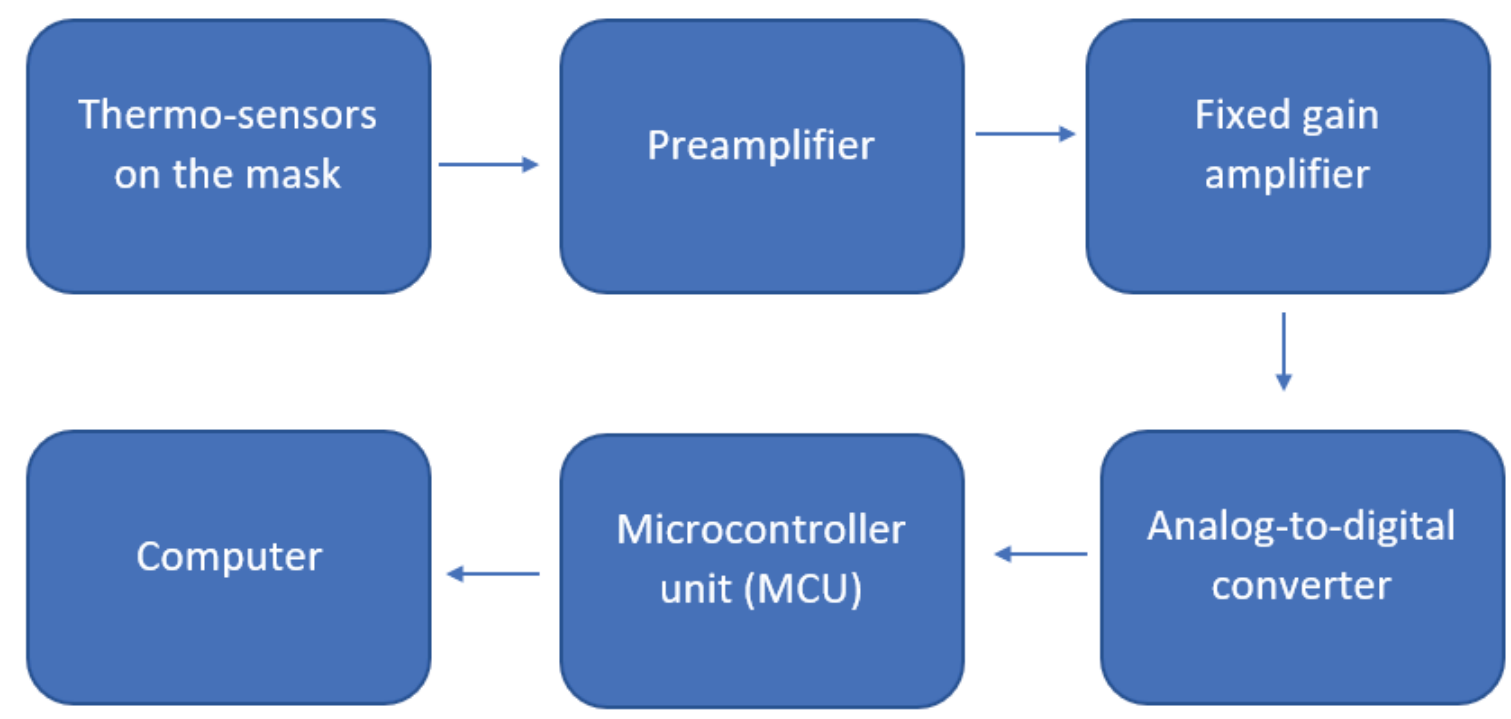

Figure 3. Block diagram of the system. 
Based on the design requirements, a measurement of six textile electrodes was selected. The jacket includes six conductive plasters detecting biopotentials in different positions in order to obtain versatile measurements. Depending on the manner in which the baby lies (or is held) and the pressure, one of the clothing surfaces is always in contact with the infant's skin. The system collects the signals continuously, and the strongest one is sent for further processing.

Several prototypes were constructed: test patches with various silver and gold textile electrodes, as well as a blanket with massive silver electrodes. The silver electrodes were made of silver-coated nylons (produced by Shieldex ${ }^{\circledR)}$. Three cotton layers were applied, and the middle one was integrated with an electrical circuit using a Shieldex ${ }^{\circledR}$ silver yarn. The electrical circuit was sewn with Shieldex ${ }^{\circledR}$ yarn on the middle layer of the textile electrode. An electrode was sewn on the first layer, stitching the electrical circuit on the middle layer. The carbon wire ends were insulated and sewn to the circuit on the middle layer. The connection between the electrode and the monitor was achieved using carbon fibers integrated with single-use gel electrodes. The third cotton layer was used for insulation and was connected to the remaining layers. Golden printed electrodes were made of thin and soft fiber with metal print designed by TNO (Eindhoven, The Netherlands). The construction of the electrodes is shown in Figure 4.

ECG was obtained using three textile electrodes in regular configuration, and the data were registered by GE Healthcare Solar ${ }^{\circledR} 8000 \mathrm{M}$. Unprocessed digital data were imported and processed using MatLab program. High- and low-pass filters were applied to remove harmonics $\geq 50 \mathrm{~Hz}$, direct current (DC) components, and high-frequency noise [18].

\subsection{Pulse sensors}

Saturation is a parameter of peripheral blood hemoglobin loading with oxygen. A pulse oximeter is used for measurement of the oxygen saturation level. Pulse oximetry enables us to recognize and evaluate the symptoms of physiological and pathological changes in infants. It enables constant

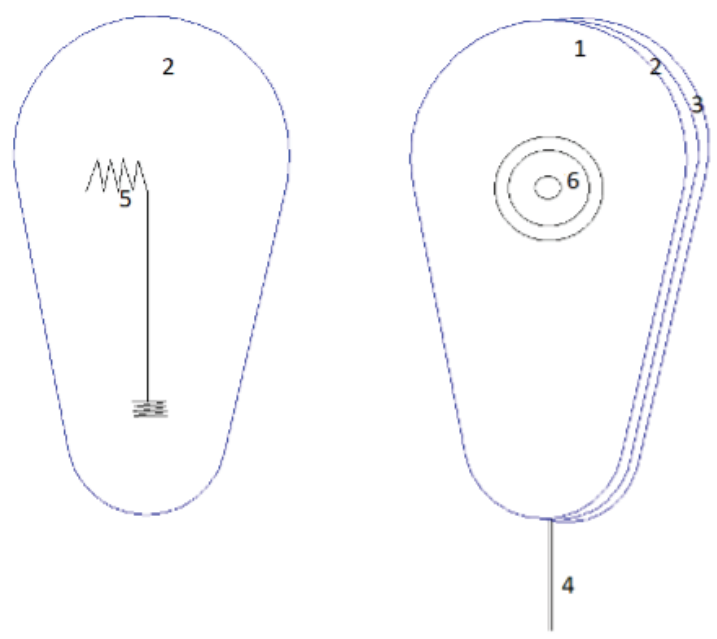

Figure 4. The construction of the textile electrodes: (1) first cotton layer, (2) second (middle) cotton layer, (3) third cotton layer, (4) carbon wires, (5) electrical circuit sewn with Shieldex yarn, and (6) gel electrode. observation of a child's life parameters and evaluation of the level of premature baby's life risk. Because of the long-term observation of saturation, monitoring should be conducted safely and noninvasively for a baby, with an easy and simpleto-use approach [20].

Yves Rimet et al. [21] created a system for wireless pulse oximetry $\left(\mathrm{SpO}_{2}\right)$ monitoring, aximetry, and children position. Sensors, electronic components, and power supply were integrated in a specially designed shoe, the BBA bootee. These baby booties have been developed based on an oximetry module OEM III (Nonin Medical Inc.) and a triaxial accelerometer (Analog Devices) to monitor many parameters in infants. Connection and data transmission from sensors are managed by the MC transceiver (Nordic semiconductor; ref. nRF9E5) through shortwave radio frequency link. The customizable straps, which are made of elastic materials, provide skin-to-skin contact for the $\mathrm{SpO}_{2}$ sensor and allow the shoe to adapt to the foot as the child grows. Owing to sensor integration with the shoe, it is possible to conduct pulse oximetry, as well as detect movement and the position of infants. All the electronic components, including power supply and its charging circuit, are assembled in a plastic case (IP64), which is built into the sole of the designed shoe. The design of the baby shoe includes a unit that receives and processes the collected data. The monitoring-and-recording unit, in the form of a box with dimensions of $15 \mathrm{~cm} \times 8 \mathrm{~cm} \times 5.5 \mathrm{~cm}$, displays the oximetry values. In addition, this unit can be programmed to give an audible and visual signal when the values exceed a threshold.

The textile support of the BBA shoe is made like normal children's clothing and is offered in several sizes. There is a hole in the sole where the pulse oximetry sensor is mounted. A single shoe is sufficient to ensure ample monitoring. The whole shoe is small and weighs $34 \mathrm{~g}$ [21].

\subsection{Humidity sensors}

The skin is a source of fluid and heat loss, and the lack of an appropriate skin barrier causes TEWL, which may contribute to dehydration and hypothermia of premature babies. The rate of water loss varies in relation to the age of the baby and is inversely related to the postpartum age. Newborns born between 24 and 25 weeks of gestation age lose an average of about $150 \mathrm{~g}$ of water per kilogram of body weight per day. TEWL is also dependent on the relative humidity of the child's environment; therefore, premature babies are placed in incubators where high air humidity is maintained [22].

An important parameter that should be controlled in preterm babies is body or underwear moisture. This factor has a significant impact on the wearing comfort and may be a symptom of the disease [23].

In order to ensure a thermoneutral environment for premature babies, appropriate temperature and humidity should be maintained around the child's body. Currently, incubators are used for placing premature babies. Severino Peixoto Nunes Netto et al. [24] are working on designing a low-cost device 
for monitoring temperature and humidity in incubators for neonates. The device displays the monitored parameters at specified intervals, along with notifications and visual alarms, allowing medical personnel to remotely access information about the infants' health. The device is controlled by the ESP8266 NodeMcu, which is an Internet of Things (IoT) platform. This system has a built-in wireless fidelity (Wi-Fi) module; it is programmed in the $\mathrm{C}++$ programming language on the Arduino board. The entire system is powered with a voltage of 3.3-5.0 V via a Universal Serial Bus (USB) cable. A DHT22 temperature-and-humidity sensor was used with an operating temperature range from $-40{ }^{\circ} \mathrm{C}$ to $80^{\circ} \mathrm{C}$, humidity in the range of $0 \%-100 \%$, and a resolution of $0.1^{\circ} \mathrm{C}$ for temperature and $2 \%-5 \%$ for humidity. The sensor contains four pins, although only three of them are used (power, ground, and data pin). The LEDs are used to warn if the parameters exceed the limit values.

Monitoring is carried out using the ThingSpeak ${ }^{\mathrm{TM}}$ Web service platform, which is an open application programming interface (API) service that works as a host for different data sent to the cloud. The sensor operation tests were conducted to ensure that the sensor was working properly, by analyzing its behavior when subjected to sudden temperature changes, such as on placement inside a fridge or near a flame. In the second stage of testing, the device was placed in a working Vision 2286 incubator (Fanem, São Paulo, Brazil). This test was conducted at the Onofre Lopes University Hospital (HUOL) of the Federal University of Rio Grande do Norte (UFRN). The main aspect analyzed under the supervision of a clinical engineer and a nurse was the refresh rate of the Web application in terms of temperature and humidity changes [24].

Jakubas et al. [23], in their textile solution for humidity measurement, used the PW109 humidity sensor, which is based on the Plug\&Wear company's sensor. It is manufactured from polyester yarn and American Iron and Steel Institute (AISI) $316 \mathrm{~L}$ stainless steel yarn with low carbon content. The sensor has a flexible structure and its dimensions are $5 \mathrm{~cm} \times 5 \mathrm{~cm}$. The humidity is measured by changing the resistance of the areas made of polyester yarn between the paths made of stainless steel thread under the influence of moisture. The humidity sensor is made in the form of a knitted fabric that

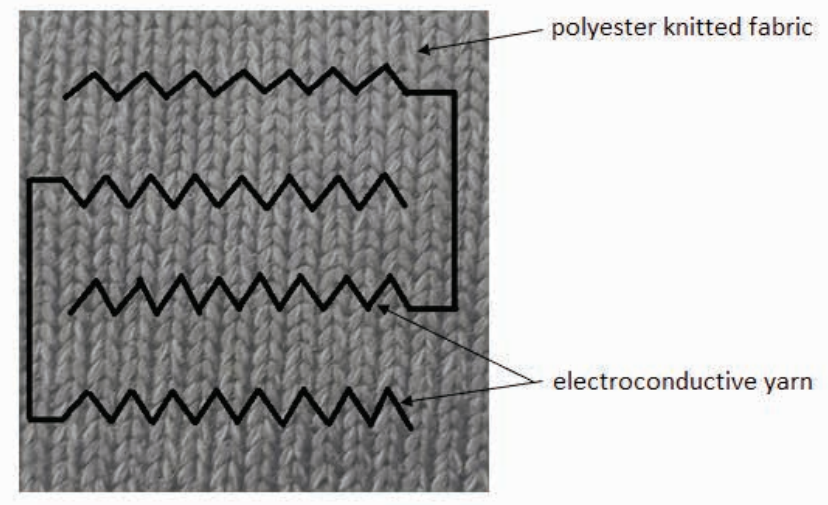

Figure 5. A schematic drawing of the moisture sensor with the areas marked. can be embedded into baby's garments. An ATmega328 microprocessor is responsible for operation of the entire system. Figure 5 shows a schematic drawing of the moisture sensor with the areas marked.

This sensor is best placed where the clothing is most exposed to moisture. The textile humidity sensor makes it possible to detect moisture, but it does not indicate the moisture content.

\section{$\underline{2.6 \text { Integrated sensor-based system }}$}

A multidisciplinary team of researchers has developed innovative technologies to facilitate the care of premature babies. Luca Piccini et al. [25] created a prototype platform that includes a photosensitive belt of textile fibers with sensors and transducers built into the belt, providing discreet monitoring of the child's breathing rate (BR), HR, temperature $(T)$, and body movement.

The belt is produced from cotton and lycra to decrease contact instability by ensuring a better fit of the sensor to the body, and it is $65 \mathrm{~cm}$ long. The belt is placed around the baby's chest and its two ends are laid on the cot. The belt can be adjusted to the size of the baby and can be regulated in a noninvasive way to prevent direct contact of the rough material with the baby's skin. The tips are squeezed together with the clasp until the band fits the size of the baby's chest. The front is powered by a pocket for external pickups. They consist of an NTC thermistor (B57550G550; Epcos, Munich, Germany) for measuring body temperature and a linear accelerometer made by using microelectromechanical systems (MEMS) technology (LIS3L06AL; STMicroelectronics, Catania, Italy) to detect the movements of the newborn.

In addition, the „BioBelt” project is an electronic unit consisting of an analog preprocessing board that receives signals provided by individual sensors on the belt and a transmission unit with a Bluetooth (BT) module for wireless data transfer to a computer outside the incubator.

To evaluate an infant's breathing, indications of chest motion and dilation amplitude are needed, without the need to calibrate the textile sensor. The MEM microchip needed active low-pass filtering to connect to a digital acquisition device. The BT transceiver used was a commercial PAN1540 module (Panasonic, Langen, Germany). The device has been designed to meet the International Electrotechnical Commission (IEC) 60601-1 standard for the safety of medical electrical devices.

A portable pulse oximeter (Radical-7; Masimo Corp., Irvine, CA, USA) was also used, which provided $\mathrm{SpO}_{2}$ and $\mathrm{HR}$ measurements. A neonatal photoplethysmography probe was applied to an infant's foot or hand to replicate a typical ICU monitoring apparatus.

The developed prototype for monitoring the biosignals of premature infants was assessed during preclinical study sessions. The study involved four subjects, who were children aged 2-12 months; it was a preliminary assessment needed for the actual setting of the clinical incubator. ECG results were 
obtained, the quality of which was reduced by the instability of the skin-belt contact as a result of infant movements. In addition, it can be assumed that the problem will be reduced during clinical trials on preterm infants in incubators, as they show poor mobility, especially in the first months of life. Further refinement of the prototype will include adjusting the sensitivity of the strain gauge to enable a more complete study of respiratory function in premature infants [25].

Carsten Linti et al. [26] developed a vest for children, which has many sensory functions. It includes completely integrated sensors for HR, respiration rate, body temperature, and humidity (through sweating). The design of the children's sensory vest has mainly focused on processes and materials that are compatible with the body and skin, as well as the textile manufacturing processes. Durability against body fluids and washing conditions was another requirement. The prototype was fabricated using weaving, sewing, knitting, printing, and coating technologies generally used in the textile industries. Signal transmission was achieved using flexible, Teflon-based insulated microconductors (American wire gauge [AWG] 36), which were woven into a narrow strip forming a textile ribbon cable. Single wires were isolated from the tape according to the location of the sensors. This tape is attached to the inside of the waistcoat and coated with another textile layer for better comfort.

The chest and abdominal breathing sensors are located on the front of the vest in the form of belts that can be fastened and adjusted. The resistive strain sensors contain a carbon-filled elastomer, which is coated on a textile ground.

For ECG measurement, dry electrodes that were placed on the inside surface of the vest composed of conductive silicone rubber printed on a textile ground were used. Conductivity was achieved using silver particles. The electrodes are located on the left and right sides of the baby's chest and abdomen, respectively.

The conductivity of the fabric is measured to determine the moisture content of the garment as a measure of the child's perspiration. Two pairs of flexible electrodes, similar to ECG electrodes, measure the moisture-dependent resistance of the textile fibers. The electrodes are located on the back of the vest.

The temperature is measured by changing the resistance of two NTC thermistors that are built into the ribbon cable. The NTC thermistors are located on the back of the vest and under the left armpit. The measuring equipment consists of a laptop; respiration, temperature, and humidity signal conditioning unit; ECG plate; and USB A/D converters. The sensor power supply is limited to $50 \mu \mathrm{A}$ each. The signals measured by the sensors are displayed on the computer screen using the LabView 5.0 application [26].

Hongyu Chen et al. [27] proposed an integrated sensor system using flexible materials. This solution consists of a vest and a cloud platform. A flexible sensor has been integrated into the structure of the vest to monitor the child's breathing, based on polydimethylsiloxane (PDMS)-graphene composite. Textile dry electrodes were used as a replacement for adhesive standard electrodes to collect the ECG signal. The vest also has built-in inertial measurement units (IMU) to get information about the movement, especially the accelerated speed of the child's wrists. When designing, materials were selected to obtain good signal quality and materials that are comfortable to use. Data on the baby's vital signs are sent to the terminal for real-time monitoring. Additionally, information from the local terminal is sent to a cloud platform, where doctors can analyze the health of premature babies. The vest is made of soft cotton containing a breath sensor based on the PDMSgraphene composite, textile electrodes for ECG measurement, and two IMUs for motion monitoring. The data acquisition and processing module consists of textile electrodes, an analog panel, an IMU, a flexible breathing sensor, an MCU, and a BT module. A breathing sensor based on the PDMS-graphene composite is located on the waist. IMU sensors are built into the wrist parts of the vest. The vest has an open structure, which makes it easy to take off and put on clothes. Velcro sewn in the sleeves and on the chest are used to stabilize the position of the sensors.

The breathing sensor is connected on one side to the power supply from the circuit and on the other side to a 12-bit A/D converter on the MSP430 (Texas Instruments, Dallas, TX, USA) MCU to ground in the circuit. The output voltage changes depending on the resistance of the sensor composed of PDMSgraphene. ECG and motion signals were separately gathered by textile electrodes and the IMU. All signal acquisition modules were controlled and managed by the $\mathrm{MCU}$ at a sampling rate of $500 \mathrm{~Hz}$. The data transfer rate in the communication process between the BT and the terminal is 92,000 bits/second. A charging circuit has been created on the board and the entire system is powered by a $3.7 \mathrm{~V}$ lithium battery. An experiment was carried out to check the operation of the integrated sensor system, and on this basis, it was found that the system can continuously monitor the baby for 8 hours using a $600 \mathrm{mAh}$ battery.

Graphene and xylene were used to create the PDMSgraphene compound. Xylene, PDMS prepolymer, curing agent, and graphene sheets were mixed in a beaker. The PMDS prepolymer was dissolved in xylene, which serves as a solvent for the dispersion of graphene in PDMS. The mixture was then subjected to bath sonication followed by stirring to widely disperse the graphene sheets. Next, the mixture was poured into a glass dish to vaporize the xylene under ambient conditions. Finally, it was cured at $65^{\circ} \mathrm{C}$ in an oven to receive the final product.

The textile electrode is disposable because sweat or other substances influence the measurement. The electrode consists of a metal clasp, conductive e-textile, and cotton. The e-textile is connected to the metal electrode latch. The cotton layer is located between the layers of e-material. The up and down layers of the e-textile are stitched by sewing.

For accurate motion measurements, an IMU unit is used, which combines accelerometers, gyroscopes, and magnetometers. 
Researchers obtained motion signals from two IMUs (MPU9250; Invensense, San Jose, CA, USA) separately for the infant's right and left wrists. The traffic data are sent to the MCU via the SPI via flexible printed circuit (FPC) wires [27].

\section{SUMMARY AND CONCLUSION}

Low birthweight of preterm babies increases the risk of health complications at the later stage of development; therefore, neonatal care is a crucial factor that should be implemented as soon as possible. The number of preterm babies does not decrease year by year, and in Poland, preterm babies constitute $6 \%-7 \%$ of all births. Maintaining children's survivability, quality of future development, and hospitalization costs are the main problems and challenges that neonatal ICUs face [28].

The purpose of this illustrative article is cognition of all important problems concerning preterm infants' health care and to enhance knowledge of innovative textronic solutions that make care and monitoring of life parameters of preterm infants easier. Solutions concerning integration of electronics with textile materials have been discussed, as well as the monitoring of life parameters such as HR, blood pressure, saturation, body temperature, respiration, and humidity.

Due to the growing demand for innovative solutions for premature babies, which support the care and constant observation of vital parameters of newborns, the authors decided to develop clothing for premature babies that would provide physiological comfort and an optimal environment for the child's development, limiting the loss of heat and water from the newborn's body. The garment for premature babies should contain compatible sensors in its structure, which monitor the life parameters of the infant and the parameters of the child's environment, thus improving the quality of life for the child and increasing the parents' sense of safety. It is assumed that parents will feel more psychologically comfortable knowing that their child is constantly monitored and protected against the effects of adverse external environment. In order to minimize mortality among premature babies, an environment in which the baby will have the lowest possible energy loss, i.e., a thermoneutral environment, should be created [29]. For this purpose, the authors decided to choose a set of monitoring sensors for the body, subclothing, and environmental temperature and humidity, as well as the vital signs, in a specially designed threelayer garment for premature babies. With this solution, it will be possible not only to provide a thermoneutral environment but also to monitor the impact of environmental conditions on the child's thermoregulation. The placement of sensors such that they do not affect the child's functional and sensory comfort and the selection of sensors that do not irritate the preterm infant's underdeveloped skin are areas that still require research. Due to the creation of an interdisciplinary field, namely, textronics, it is possible to constantly monitor the state of life of premature babies through the use of sensors in clothing for everyday use. The development of biomedicine, wireless communication, and information systems has contributed to the creation of a new tool for monitoring the vital parameters of premature children, which is of increasing interest among scientists and clinicians [9]. Due to the miniaturization and low weight of the sensors, it is easier to integrate them with clothing or accessories (also from an ergonomic point of view) [30].

Recently, significant progress has been made in the area of monitoring of preterm infants toward noninvasive monitoring of vital signs. A necessary step is to improve the sensors for signal processing and integration to minimize the occurrence of motion artifacts, design sensors with higher sensitivity, and optimize sensor integration. The biggest challenge is the miniaturization of devices and their power supply, as well as minimizing the occurrence of various types of undesirable artifacts [18].

Textile electrodes are a new, attractive solution for biopotential measurements. Some scientists have used polyurethane fabrics coated with metal, usually silver or gold, in their textile electrode solutions. These special electrodes are made of fabric with the appearance of an electrode. Essentially, textiles are insulators, while in textile electrodes, conductive yarns are woven, knitted, or embroidered into the fabric during the manufacturing process. The textile electrodes have a knitted structure with a metal part woven into the material. However, it is worth paying attention to the fact that textile electrodes have some disadvantages. Textile electrodes with a knitted or woven structure are usually of poor quality and their contact with the skin is feeble. Additionally, the process of washing textile electrodes poses a problem. Therefore, it is required to develop methods of attaching textile electrodes to the material and to optimize the measurement methods [9].

Sensors for monitoring the health of premature babies have a wide range of applications, but it should be remembered that there are issues that require improvement and further research. Such issues as the reliability of vital signs' detection, the minimization of artifacts, the use of reliable and long-lasting power supplies, and the extension of wireless communication range require improvements. Sensors should be energy-saving and cheap to be widely used in everyday life [9].

When designing a device for monitoring the physiological parameters of premature babies, it is necessary to take into account the comfort of use, appearance, size, and technical aspects, such as ensuring an accurate and continuous measurement of vital functions, the possibility of disinfection, and safety in use. Apart from ensuring the comfort of use, the material from which the clothes for premature babies are made should also be safe and breathable [15].

\section{Acknowledgement}

These studies were financed from funds assigned from: 142/501-4-42-1-1 statutory activity by the Lodz University of Technology. Institute of Material Science of Textiles and Polymer Composites. Poland. 


\section{References}

[1] WHO. (2020). Web site: https://www.who.int/newsroom/fact-sheets/detail/preterm-birth. (Accessed on 20.08.2020).

[2] Bomba-Opoń, D., Wilegoś, M. (2016). Standardy opieki okołoporodowej w przypadkach występowania określonych powikłań. Ginekologia i Perinatologia Praktyczna, 1(1), 1-9.

[3] Rocznik demograficzny (2019). str. 287, tabela 79.

[4] Łada-Tondyra, E., Jakubas, A. (2018). Nowoczesne zastosowanie systemów tekstronicznych. Przegląd elektrotechniczny, ISNN 0033-2097,R.94, 94 NR 12/2018.

[5] Zięba, J., Frydrysiak, M. (2006). Textronics - electrical and electronic textiles. Sensors for breathing frequency measurement. Fibres \& Textiles in Easterm Europe, 14(5), 59.

[6] Furtak, N. T., Skrzetuska, E., Krucińska, I. (2013). Development of screen-printed breathing rate sensors. Fibres \& Textiles in Eastern Europe, 21, 6(102), 84-88.

[7] Jakubas, A., Łada-Tondyra, E. (2017). Analiza ściegów ściągaczowych jako czujników rytmu oddechowego, Zeszyty Naukowe Wydziału Elektroniki i Automatyki Politechniki Gdańskiej Nr 54, XLIX Międzyuczelniana Konferencja Metrologów MKM 2017.

[8] Jakubas, A., Łada-Tondyra, E., Nowak, M., Margol, M., Lipińska-Opałka, A. (2015). Koncepcja tekstronicznego systemu do pomiarów funkcji życiowych małych dzieci; PRZEGLĄD ELEKTROTECHNICZNY, ISSN 0033-2097, R. 91 NR 12/2015, doi: 10.15199/48.2015.12.30.

[9] Zhu, Z., Liu, T., Li, G., Li, T., Inoue, Y. (2015). Wearable sensor systems for infants. Sensors, 15, 3721-3749. doi:10.3390/s150203721.

[10] Rozalska-Walaszek, I., Lesiuk, W., Aftyka, A., Lesiuk, L. (2012). Opieka pielęgniarska nad wcześniakiem leczonym na oddziale intensywnej terapii noworodka. Nursing Topics, 20(3), 409-415.

[11] Krucińska, I., Skrzetuska, E., Kowalski, K. (2019). Application of a thermal mannequin to the assessment of the heat insulating power of protective garments for premature babies. Autex Research Journal, 19(2), 134146. doi: 10.1515/aut-2018-0010.

[12] Gulczyńska, E., Cedrowska-Adamus, W. (2018). Prewencja hipotermii jatrogennej u noworodków urodzonych przedwcześnie. Postępy Neonatologii, 24(2), 123-127. doi: 10.31350/postepyneonatologii/2018/2/ PN2018023.

[13] Kumar, N., Akangire, G., Sullivan, B., Fairchild,K., Sampath, V. (2020). Continuous vital sign analysis for predicting and preventing neonatal diseases in the twentyfirst century: Big data to the forefront. Pediatric Research, 87, 210-220. doi: 10.10 38/s41390-019-0527-0.

[14] Farahani, H., Wagiran, R., Hamidon, M. Z. (2014). Humidity sensors principle, mechanism, and fabrication technologies: A comprehensive review. Sensors, 14, 7881-7939. doi:10.3390/s140507881.

[15] Chen, W., Dols, S., Oetomo, S. B., Feijs, L. (2010). Monitoring body temperature of newborn infants at neonatal intensive care units using wearable sensors. BodyNets'2010, September 10-12, 2010, Corfu Island, Greece. Copyright 2010 ICST 978-963-9799-41-7.
[16] Cao, H., Hsu, L.-C., Ativanichayaphong, T., Sin, J., Stephanou, H. E., et al. (2007). An infant monitoring system using $\mathrm{CO}_{2}$ sensors. 2007 IEEE International Conference on RFID Gaylord Texan Resort, Grapevine, TX, USA March 26-28, 2007.

[17] Hsu, C.-H., Chow, J. C. (2005). Design and clinic monitoring of a newly developed non-attached infant apnea monitor. Biomedical Engineering: Applications, Basis and Communication, 17(3), 126-134.

[18] Bouwstra, S., Chen, W., Feijs, L., Oetomo, S. B. (2009). Smart jacket design for neonatal monitoring with wearable sensors, 2009 Body Sensor Networks.

[19] Hertleer, C., Grabowska, M., Van Langenhove, L., Catrysse, M., Hermans, B., et al. (2004). Towards a smart suit. "Wearable Electronic and Smart Textiles" Leeds, UK, 11th June 2004.

[20] Czajkowska, M., Pierzak-Sominka, J., Czajkowski, A. (2016). Application of pulse oximetry in neonatology. Problems of Applied Sciences, 4, 121-124.

[21] Rimet, Y., Brusquet, Y., Ronayette, D., Dageville, C., Lubrano, M., et al. (2007). Surveillance of infants at risk of apparent life threatening events (ALTE) with the BBA bootee: A wearable multiparameter monitor.

[22] Ågren, J., Sjörs, G., Sedin, G. (2005). Ambient humidity influences the rate of skin barrier maturation in extremely preterm infants, doi: 10.1016/j.jpeds.2005.11.027, [Elsevier 2005].

[23] Jakubas, A., Łada-Tondyra, E., Nowak, M. (2017). Textile sensors used in smart clothing to monitor the vital functions of young children. 978-1-5386-1528-7/17/\$31.00 (02017 IEEE.

[24] Netto, S. P. N., Chacon, P. F. S., de Morais Junior, A. M. C., de Rubim Costa, C. L. B., Brasil, F. L. (2019). Remote monitoring of temperature and humidity - $A$ reliable and inexpensive device development applied in neonatal incubators. In: Costa-Felix, R., et al. (Eds.). XXVI Brazilian Congress on Biomedical Engineering, IFMBE Proceedings 70/1. Springer Nature Singapore Pte Ltd. doi: 10.1007/978-981-13-2119-1_106.

[25] Piccini, L., Ciani, O., Grönvall, E., Marti, P., Andreoni, G. () New monitoring approach for neonatal intensive care unit.

[26] Linti, C., Horter, H., Osterreicher, P., Planck, H. (2006). Sensory baby vest for the monitoring of infants. Proceedings of the International Workshop on Wearable and Implantable Body Sensor Networks (BSN'06) 0-76952547-4/06 \$20.00 @ 2006 IEEE.

[27] Chen, H., Bao, S., Lu, C., Wang, L., Ma, J., et al. (2020). Design of an integrated wearable multi-sensor platform based on flexible materials for neonatal monitoring. IEEE Acces, 8. doi: 10.1109/ACCESS.2020.2970469.

[28] NAUKAWPOLSCE (2008). Web site: https://naukawpolsce. pap.pl/aktualnosci/news\%2C205572\%2Cw-polskichszpitalach-brakuje-oddzialow-dla-wczesniakow.html. (Accessed on 09.2020).

[29] Netto, S. P. N., Chacon, P. F. S., de Morais Junior, A. M. C., de Rubim Costa, C. L. B., Brasil, F. L. (2019). Remote monitoring of temperature and humidity $-A$ reliable and inexpensive device development applied in neonatal incubators. In: Costa-Felix, R., et al. (Eds.). XXVI Brazilian Congress on Biomedical Engineering, IFMBE Proceedings 70/1. Springer Nature Singapore Pte Ltd. doi: 10.1007/978-981-13-2119-1_106. 
AUTEX Research Journal, DOI 10.2478/aut-2021-0034

[30] Chen, H., Xue, M., Mei, Z., Oetomo, S. B., Chen, W. (2016).

A review of wearable sensor systems for monitoring body movements of neonates. Sensors, 16, 2134. doi: 10.3390/ s16122134. Web site: www.mdpi.com/journal/sensors. 